\title{
Active Vector Separation Using Induced Charge Electro-osmosis with Polarizable Obstacle Arrays
}

\author{
Hideyuki Sugioka* \\ Department of Mechanical Systems Engineering, \\ Shinshu University, 4-17-1 Wakasato, Nagano 380-8553, Japan
}

(Dated: July 22, 2016)

\begin{abstract}
Vector separation using obstacle post arrays is promising for various microfluidic applications. Here, we propose a novel active sieve using induced charge electro-osmosis (ICEO). By the multiphysics simulation technique based on the boundary element method combined with a thin electric double-layer approximation, we find that the active sieve having a polarizable post array shows excellent vector separation with dynamic size selectivity owing to the hydrodynamic interactions between the polarizable post array and the target particle. We consider that our separation device is useful for realizing innovative high-throughput biomedical systems with a simple structure.
\end{abstract}

PACS numbers:

${ }^{*}$ Electronic address: hsugioka@shinshu-u.ac.jp 


\section{INTRODUCTION}

The vector separation technique using obstacle post arrays in a microfluidic channel has been attracting much attention since it enables continuous rapid separation with a simple mechanism [1-4]. Here, vector separation means the separation that changes the direction of flow in different ways based on the characteristics of a target particle. For example, Ertas theoretically showed that because of a broken reflection symmetry in the obstacle design, the direction of the motion of the target particle in the sieve varies as a function of its self-diffusion [1]. Huang et al. experimentally showed that the target particle chooses its path deterministically on the basis of its size in the obstacle post array [2]. Balvin et al. showed that the motion of spheres is irreversible and displays directional locking in the obstacle arrays [3]. Furthermore, MacDonald et al. proposed a microfluidic sorting device using an optical lattice and demonstrated that it shows $45^{\circ}$ angular separation [5]. Obviously, this active vector separation method has a large merit and is attractive since it provides dynamic selectivity for various physical parameters of target particles owing to its separation mechanism along with the possibility of preventing the so-called fouling or clogging problem [4], which is essential for commercial viability. Nevertheless, to the best of our knowledge, the active vector separation method using obstacle post arrays has not been explored well. Thus, the study of the active vector separation method is important for promising microfluidic applications. However, since the optical lattice uses a high-power laser beam (e.g., $530 \mathrm{~mW}$ ) [5], it requires large equipment even though the microfluidic chip is very small. Thus, simpler methods are required for the total miniaturization of the systems. In addition, induced charge electro-osmosis (ICEO) has recently been attracting much attention because of its high possibility for various biomedical applications $[6,7]$ since it provides large flow velocities $(\sim 1 \mathrm{~mm} / \mathrm{s})$ at small ac applied voltages $(\sim 1 \mathrm{~V})$ with simple structures. Here, ICEO is the electrokinetic flow phenomena that produce large quadrupolar vortex flows around a polarizable particle because of the interaction between the tangential electric field and the induced electric double layer on the polarized particle. Thus, ICEO is probably suitable for realizing a miniaturized active vector separation device system. Therefore, here, we propose a novel active sieve using the hydrodynamic interactions between a polarizable obstacle post array and a polarizable target particle on the basis of ICEO. Namely, in this manuscript, we focus on the development of the active vector separation method and 


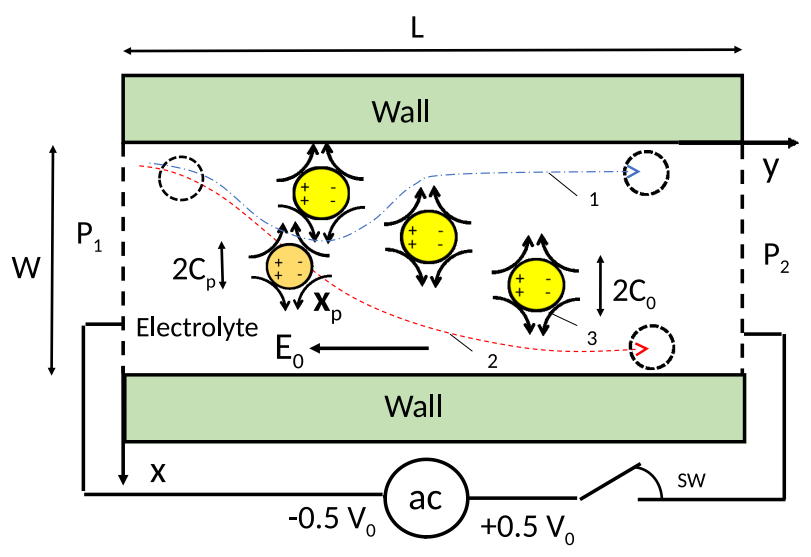

FIG. 1: (Color online) Schematic view of active sieve using induced charge electro-osmosis (ICEO). 1: passing mode. 2: separation mode. 3: ICEO vortex flow. Here, length $L=2.25 w$ and width $w$ $=100 \mu \mathrm{m}$; the positions $\left(x_{i}, y_{i}\right)$ of the polarizable particle $i$ are typically $(0.2,0.725),(0.4,1.125)$, and $(0.6,1.525)$ for $i=1-3$, respectively, whereas the initial position of the target particle $\left[\left(x_{p}, y_{p}\right)\right.$ at $t=0]$ is typically $(0.2,0.325)$.

elucidate its design concept.

\section{THEORY}

Figure 1 shows a schematic view of the proposed active sieve using ICEO. As shown in Fig. 1, we consider a polarizable obstacle array having a line of three conductive circular cylinders of radius $c_{0}(=0.1 w)$ and a two-dimensional $(2 \mathrm{D})$ target particle of radius $c_{p}$ (0.01 to $0.15 w)$ in a rectangular channel of length $L(=2.25 w)$ and width $w(=100 \mu \mathrm{m})$. In Fig. 1, the positions $\left(x_{i}, y_{i}\right)$ of the polarizable particle $i$ are typically $(0.2,0.725),(0.4$, $1.125)$, and $(0.6,1.525)$ for $i=1,2$, and 3 , respectively, whereas the initial position of the target particle $\left[\left(x_{p}, y_{p}\right) \equiv\left(x_{0}, y_{0}\right)\right.$ at $\left.t=0\right]$ is typically $(0.2,0.325)$. Furthermore, the pressures at the inlet and outlet positions are $p_{1}$ and $p_{2}\left(>p_{1}\right)$, whereas the electric potentials at the inlet and outlet positions can be assumed to be $-V_{0} / 2$ and $+V_{0} / 2$ without loss generality, respectively. Namely, in this device, we can apply ac voltages of amplitude $V_{0}$ and a pressure difference $\Delta p\left(=p_{2}-p_{1}>0\right)$ between the inlet and outlet positions as a control parameter of the separation device. That is, the trajectory of the target particle 
can be changed from a passing mode to a separation mode by applying a parallel electric field $E_{0}\left(\equiv V_{0} / L\right)$ in the $-y$-direction, as shown in Fig. 1. In other words, since the target particle experiences a repulsive force in the $x$-direction from the first conductive post at $\left(x_{1}, y_{1}\right)=(0.2,0.725)$, the polarizable particle under a sufficient parallel electric field cannot pass the gap region between the first and second conductive posts and thus this device can provide the vector separation of different particles. Note that the hydrodynamic interactions between polarizable particles due to ICEO have been discussed in detail, e.g., in Ref. [8].

Furthermore, the multi-physics calculation method used here is almost the same as that in Ref. [9], except that, here, we consider the hydrodynamic interactions between the fixed polarized array and the movable polarizable particle. Thus, please see Ref. [9] for details. However, for the reader's convenience, we here briefly explain the calculation method; i.e., we assume non-Brownian Stokes flow at a low Reynolds number, and basis of the thin double-layer approximation, we solve the Stokes equations

$$
\mu \nabla^{2} \boldsymbol{v}-\nabla p=0, \quad \nabla \cdot \boldsymbol{v}=0
$$

under the boundary condition that

$$
\boldsymbol{v}^{(j)}=\boldsymbol{U}^{(j)}+\boldsymbol{\Omega}^{(j)} \times \boldsymbol{x}^{(j)}+\boldsymbol{v}_{\mathrm{s}}^{(j)}
$$

on the surfaces of both the obstacle post $(j=1,2,3)$ and the target particle $(j=0)$ with the additional force and torque balance conditions (that consider a Maxwell stress), where $\mu(\sim 1 \mathrm{mPa} \cdot \mathrm{s})$ is the fluid viscosity, $\boldsymbol{v}$ is the velocity, $p$ is the pressure, and $\boldsymbol{U}^{(j)}$ and $\boldsymbol{\Omega}^{(j)}$ are the translational and rotational angular velocities, respectively. Furthermore, $\boldsymbol{v}_{s}^{(j)}$ is the ICEO slip velocity and it is described using the Helmholtz-Smoluchowski formula; of course, $\boldsymbol{U}^{(j)}$ and $\boldsymbol{\Omega}^{(j)}$ are zero on the surface of the obstacle particles.

\section{RESULTS}

Figure 2 shows the time evolution of the flow fields of the active vector separation using ICEO under the condition that $\Delta p=4$ Pa. As shown in Figs. 2(a)-2(c), a target particle of radius $c_{p} / w=0.1$ shows the passing mode at $V_{0}=0 \mathrm{~V}$, whereas the particle shows the separation mode at $V_{0}=3 \mathrm{~V}$, as shown in Figs. 2(d)-2(f). More specifically, as shown in Fig. 2(b), the target particle at $V_{0}=0 \mathrm{~V}$ can pass through the gap region between the first 


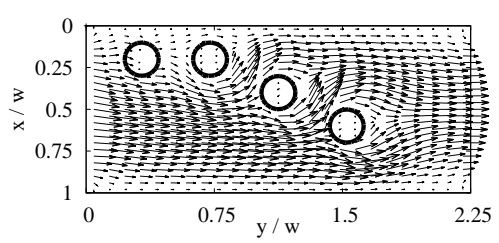

(a) $t / T_{0}=0\left(V_{0}=0 \mathrm{~V}\right.$, $\left.c_{p} / w=0.1\right)$

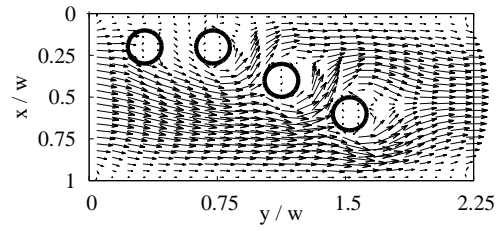

(d) $t / T_{0}=0\left(V_{0}=3 \mathrm{~V}\right.$, $\left.c_{p} / w=0.1\right)$

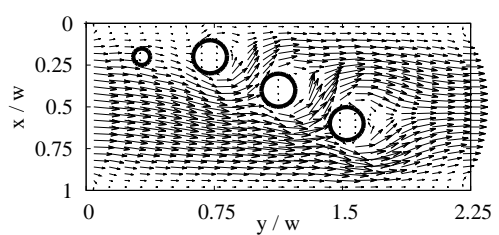

(g) $t / T_{0}=0\left(V_{0}=3 \mathrm{~V}\right.$, $\left.c_{p} / w=0.05\right)$

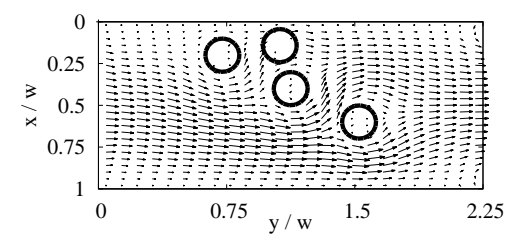

(b) $t / T_{0}=50\left(V_{0}=0 \mathrm{~V}\right.$,

$\left.c_{p} / w=0.1\right)$

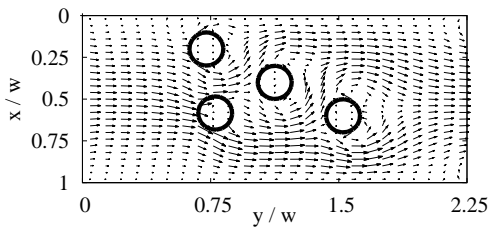

(e) $t / T_{0}=25\left(V_{0}=3 \mathrm{~V}\right.$,

$\left.c_{p} / w=0.1\right)$

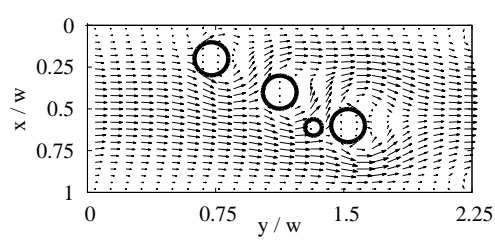

(h) $t / T_{0}=52\left(V_{0}=3 \mathrm{~V}\right.$,

$\left.c_{0} / w=0.05\right)$

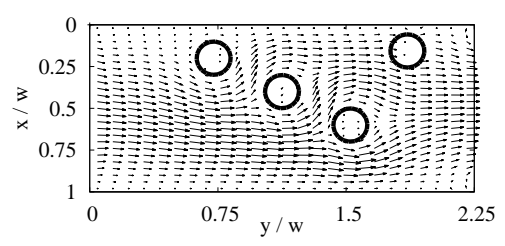

(c) $t / T_{0}=77\left(V_{0}=0 \mathrm{~V}\right.$, $\left.c_{p} / w=0.1\right)$

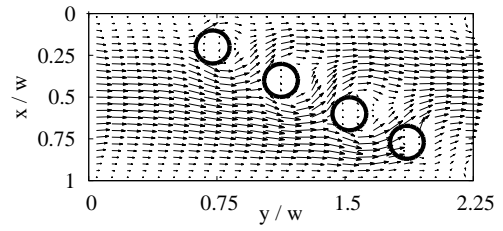

(f) $t / T_{0}=55\left(V_{0}=3 \mathrm{~V}\right.$, $\left.c_{p} / w=0.1\right)$

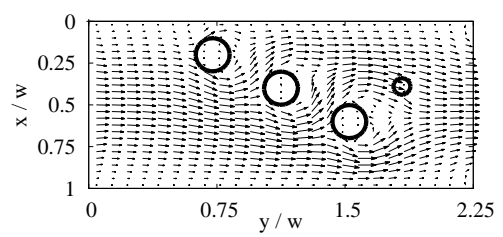

(i) $t / T_{0}=64\left(V_{0}=3 \mathrm{~V}\right.$, $\left.c_{p} / w=0.05\right)$

FIG. 2: Flow fields of the active separation. Here, $L_{0} / w=2.25, c_{0} / w=0.1, w=100 \mu \mathrm{m}, \Delta P=4$ $\mathrm{Pa}, \mu=1 \mathrm{mPa}$ s, and $T_{0}=1 \mathrm{~ms}$.

and second posts owing to the surrounding flow of the first post, whereas the target particle at $V_{0}=3 \mathrm{~V}$ is subjected to the ICEO repulsion force from the first obstacle and thus it passes through a far region below the first post, as shown in Fig. 2(e). Thus, we find that our proposed device works well, as considered in Sec. 2. Furthermore, Figs. 2(g)-2(i) show that a small target particle of radius $c_{p} / w=0.5$ can pass through the gap region between the second and third posts. This is because the repulsive force of the small particle $\left(c_{p} / w=0.5\right)$ is much smaller than that of the large particle $\left(c_{p} / w=0.5\right)$ since the ICEO flow velocity is proportional to the radius of the particle. Note that the ICEO-represented slip velocity $[6,7]$ is described as

$$
U_{0}=\frac{\epsilon c_{p} E_{0}^{2}}{\mu},
$$

although it is a solution for an unbounded problem of ICEO. 


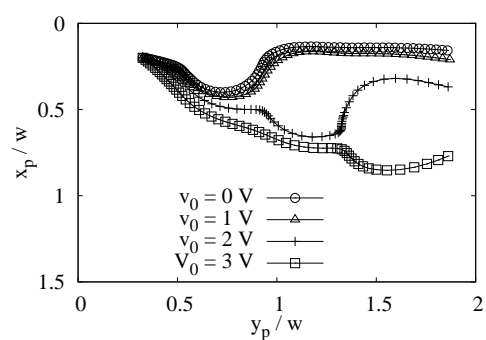

(a) Voltage dependence

$\left(c_{p} / w=0.1, v_{0}=3 \mathrm{~V}, \Delta P=4\right.$

$\mathrm{Pa})$.

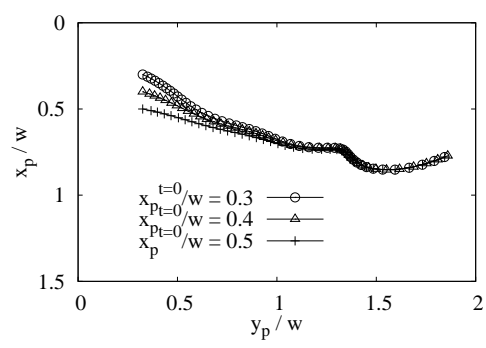

(d) Initial position dependence when $c_{p} / w=0.1, v_{0}=3 \mathrm{~V}$, and

$$
\Delta P=4 \mathrm{~Pa} .
$$

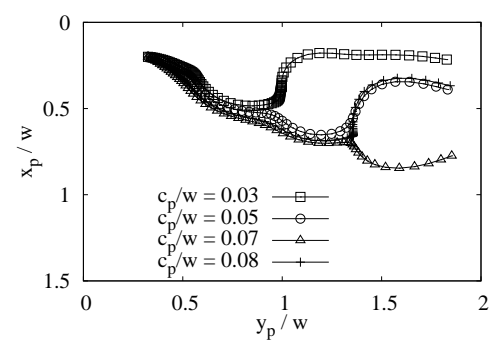

(b) Size dependence $\left(c_{p} / w=0.05\right.$, $\left.v_{0}=3 \mathrm{~V}, \Delta P=4 \mathrm{~Pa}\right)$.

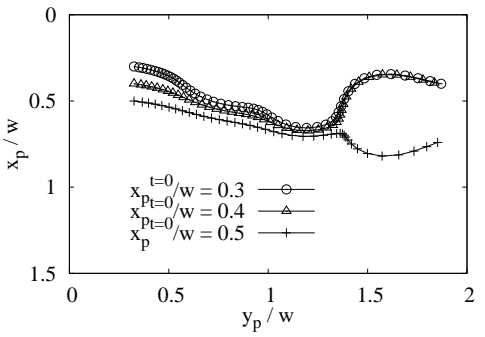

(e) Initial position dependence

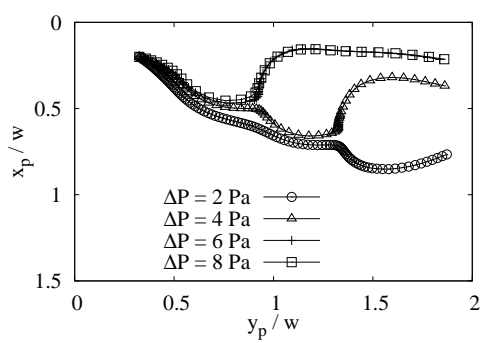

(c) Pressure dependence $\left(c_{p} / w=0.1, v_{0}=2 \mathrm{~V}, \Delta P=8\right.$

$\mathrm{Pa})$.

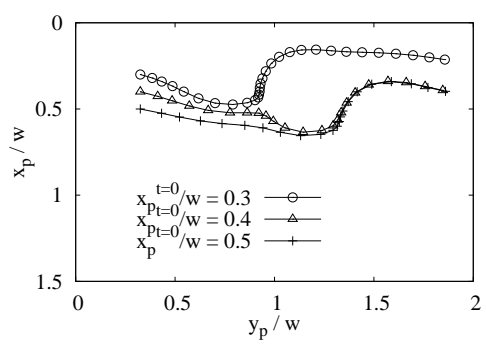

(f) Initial position dependence when $c_{p} / w=0.05, v_{0}=3 \mathrm{~V}$, and $\quad$ when $c_{p} / w=0.1, v_{0}=2 \mathrm{~V}$, and

$$
\Delta P=4 \mathrm{~Pa} .
$$$$
\Delta P=8 \mathrm{~Pa} .
$$

FIG. 3: Particle trajectories for vector separation. Here, $L_{0} / w=2.25, c_{0} / w=0.1, w=100 \mu \mathrm{m}$, $\mu=1 \mathrm{mPa} \mathrm{s}$, and $T_{0}=1 \mathrm{~ms}$.

Figure 3 shows particle trajectories for vector separation under various conditions for $V_{0}, c_{p}$, and $\Delta p$; the figure clearly shows remarkable active vector separation owing to the difference in $V_{0}, c_{p}$, and $\Delta p$. More specifically, as shown in Fig. 3(a), the particle at $V_{0}=0$ and $1 \mathrm{~V}$ passes through the first gap between the first and second posts, while the particle at $V_{0}=2$ and $3 \mathrm{~V}$ passes through the second gap between the second and third posts and the third gap between the third post and the wall. Namely, we find that the separation angle can be controlled by adjusting the applied voltage. Furthermore, as shown in Fig. 3(b), the particles at $c_{p} / w=0.03$ and $0.08 \mathrm{~V}$ pass through the first and third gaps, respectively, while the particles at $c_{p} / w=0.05$ and $0.07 \mathrm{~V}$ pass through the second gap. Namely, we find that particles of different radii can show different separation angles. Furthermore, as shown in Fig. 3(c), the particle passes through the first gap at $\Delta p=6$ and $8 \mathrm{~Pa}$, while the particle at $\Delta p=2$ and $4 \mathrm{~Pa}$ passes through the third and second gaps, respectively. Namely, we find 


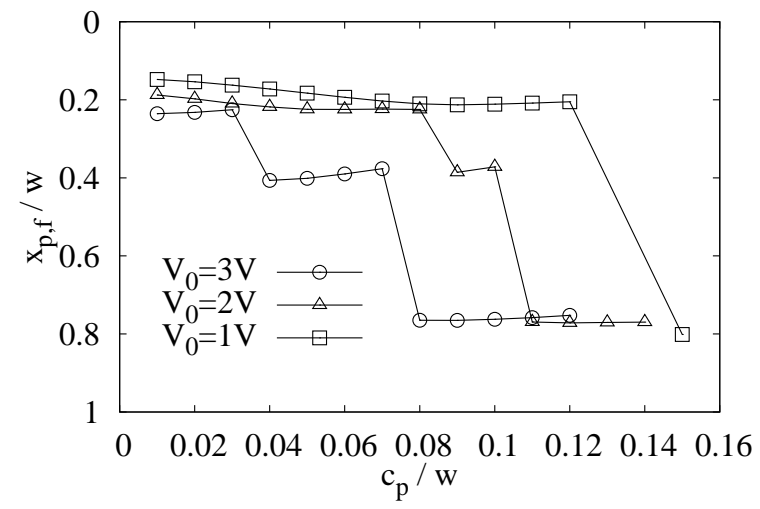

(a) Dependence of $x_{p, f}$ on $c_{p}$ at $V_{0}=1,2$, and 3

$$
(\Delta p=4 \mathrm{~Pa}) .
$$

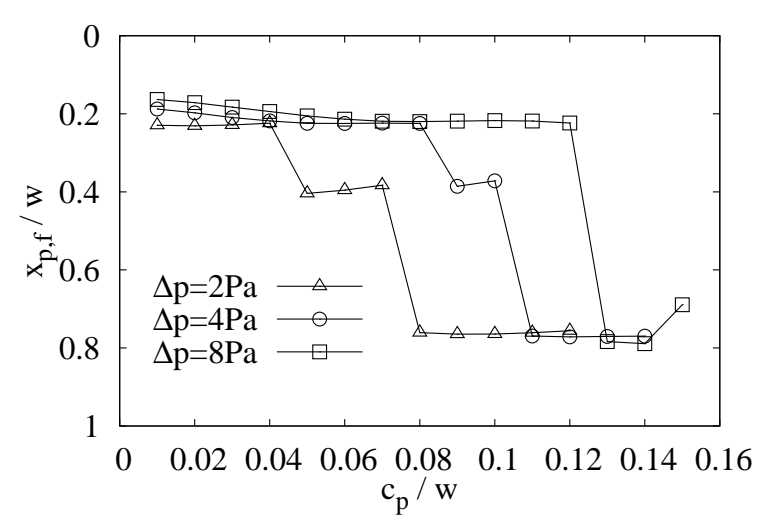

(b) Dependence of $x_{p, f}$ on $c_{p}$ at $\Delta p=2,4$, and 8 (

$$
\left.V_{0}=2 \mathrm{~V}\right)
$$

FIG. 4: Dynamic size selectivity of the active sieve using obstacle post arrays with ICEO phenomena. Here, $L_{0} / w=2.25, c / w=0.08, w=100 \mu \mathrm{m}, \Delta P=4 \mathrm{~Pa}, \mu=1 \mathrm{mPa}$ s, and $T_{0}=1$ ms.

that the separation angle can also be controlled by the applied pressure difference. This is because the separation angle is determined by adjusting the pressure balance between the ICEO repulsive pressure and the applied pressure difference. In addition, Figs. 3(d)-3(f) show that the trajectories of the active sieve using ICEO are almost locked except those of the transient condition near the threshold, similar to the ordinary passive non-Brownian deterministic sieve [3].

Figure 4 shows the dynamic size selectivity of the active sieve using obstacle post arrays with ICEO phenomena. Specifically, on the one hand, Fig. 4(a) shows the dependence of the final $x$ position of the target particle $\left(x_{p, f}\right)$ on the particle radius $\left(c_{p}\right)$ at $V_{0}=1,2$, and $3 \mathrm{~V}$ under the condition that $\Delta p=4 \mathrm{~Pa}$. As shown in Fig. 4(a), we find that the final positions of the particle are clearly separated by the difference in the radius, and the threshold size can be controlled by adjusting the applied ac voltages at a constant pressure difference. On the other hand, Fig. 4(b) shows the dependence of $x_{p, f}$ on $c_{p}$ at $\Delta p=2,4$, and $8 \mathrm{~V}$ under the condition that $V_{0}=2 \mathrm{~V}$. As shown in Fig. 4(a), we also find that the final positions of the particle are clearly separated by the difference of the radius and the threshold size can be controlled by the applied pressure difference at the constant voltage. 


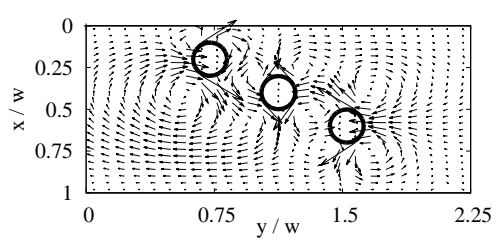

(a) Flow field with the sieve $\left(\Delta P=0 \mathrm{~Pa}, V_{0}=3 \mathrm{~V}\right)$.

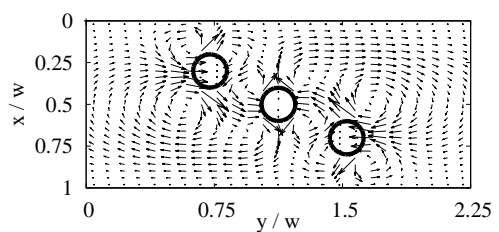

(d) Flow fields for a symmetrical sieve $\left(V_{0}=3 \mathrm{~V}\right)$.

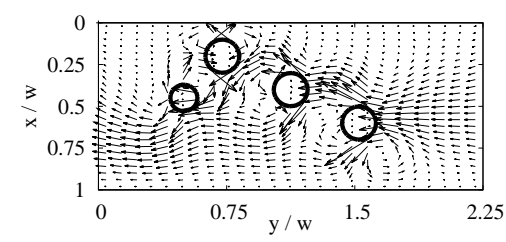

(b) Flow field with the sieve and target $\left(\Delta P=0 \mathrm{~Pa}, V_{0}=3 \mathrm{~V}\right.$, $\left.c_{p} / w=0.1\right)$.

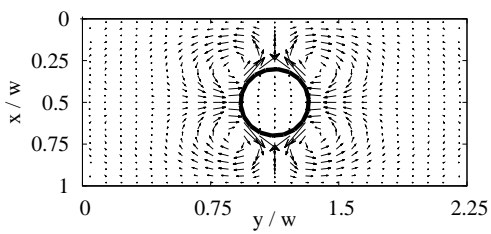

(e) Fundamental ICEO flow

$$
\left(V_{0}=3 \mathrm{~V}, c_{0} / w=0.2\right) .
$$

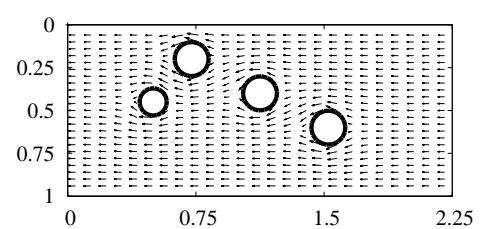

(c) Electric field with the sieve and target $\left(\Delta P=0 \mathrm{~Pa}, V_{0}=3 \mathrm{~V}\right.$, $\left.c_{p} / w=0.1\right)$.

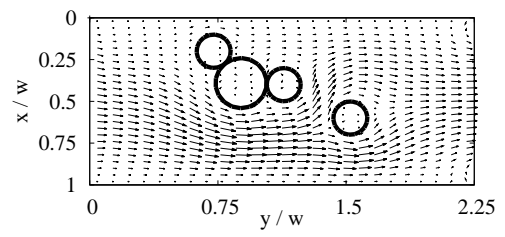

(f) Clogging phenomenon

$\left(t / T_{0}=200, V_{0}=0 \mathrm{~V}\right.$, $\left.c_{p} / w=0.15\right)$.

FIG. 5: Various physical aspects behind the active sieve and related phenomena. Here, $L_{0} / w=$ $2.25, c / w=0.08, w=100 \mu \mathrm{m}, \Delta P=4 \mathrm{~Pa}, \mu=1 \mathrm{mPa} \mathrm{s}$, and $T_{0}=1 \mathrm{~ms}$.

\section{DISCUSSION}

In Fig. 5, we summarize various physical aspects behind the active sieve and related phenomena. Specifically, Figs. 5(a) and 5(b) show the flow fields around the polarizable obstacle array with and without the polarizable target particle, respectively, under the condition that $\Delta P=0 \mathrm{~Pa}$. Of course, we cannot observe active sieve phenomena at $\Delta P=0$ $\mathrm{Pa}$ since the active vector separation phenomenon results from the pressure balance between the ICEO repulsive pressure and the applied pressure difference. However, Figs. 5(a) and 5(b) clarify the complex flow field phenomena behind the active sieve phenomena. Namely, as shown in Fig. 5(a), the flow field around the polarizable obstacle array is slightly different from that of the ordinary symmetrical ICEO vortex flow [such as a flow in Fig. 5(e)]; i.e., in Fig. 5(a), the vortex flow on the left-hand side around the first post is much stronger than that in the right-hand side, whereas the vortex flow on the right-hand side around the third post is much stronger than that on the left-hand side. This is because the constant potential boundaries at $y=0$ and $L$ affect the first and third obstacle posts and form asymmetrical 
zeta potentials on the surface of these posts. Furthermore, because of the broken symmetry at $x=0.5$, a net flow in the $-x$-direction is observed in Fig. 5(a). This phenomenon is interesting from the viewpoint of the development of microfluidic pumps [10]; however, this is beyond the scope of this study. Furthermore, as shown in Fig. 5(b), the target particle generates a more complex flow field than that shown in Fig. 5(a), although the electric field is rather simple, as shown in Fig. 5(c). That is, behind the active sieve phenomena, there is a more complex mechanism than the rather simple mechanism described in Sec. 2 and its analytical understanding will be a challenging problem in the future, although this is also beyond the scope of this study. In addition, we can consider a post array geometry with rotational symmetry, as shown in Fig. 5(d). However, it is clearly not suitable for continuous vector separation since the large gap between the first post and the upper wall may cause an error in passing, whereas the small gap between the third post and the lower wall may cause the clogging problem. Thus, an asymmetrical post array geometry is needed for the active sieve using ICEO. Furthermore, Fig. 5(f) shows a clogging phenomenon under the condition that $V_{0}=0 \mathrm{~V}$ and $c_{p} / w=0.15$. In fact, the passive sieve using an obstacle array at $V_{0}=0$ often causes the clogging problem, and because of this problem, we cannot systematically obtain the calculation results for the dependence of $x_{p, f}$ on $c_{p}$ at $V_{0}=0$. However, this fact clearly shows that the active sieve has a large merit, that is, it effectively prevents the clogging problem with a simple mechanism. Thus, our proposed device is promising; in the future, we would like to examine the phenomena experimentally.

\section{CONCLUSIONS}

We have proposed an active sieve using the hydrodynamic interactions between a polarizable obstacle array and the polarizable target particle through ICEO phenomena and numerically examined its characteristics. In particular, by the multi-physics calculation method based on the boundary element method combined with a thin double-layer approximation, we find that (1) the active sieve using ICEO phenomena shows vector separation with dynamic size selectivity by changing the applied voltage. (2) The dynamic size selectivity results from the hydrodynamic force balance between the pressure origin and ICEO forces; thus, the threshold size for separation is dependent on the pressure difference and the applied voltages. (3) The trajectories are almost locked independent of the initial position in 
the non-Brownian low-Reynolds-number flow fields and change their direction dynamically upon applying an electric voltage. We consider that the active sieve using ICEO provides a new simple mechanism for promising microfluidic separation technologies.

\section{Acknowledgments}

This work was partially supported by JSPS KAKENHI Grant Number 16K05650.

[1] D. Ertas, Phys. Rev. Lett. 80, 1548 (1998).

[2] L. R. Huang, E. C. Cox, R. H. Austin, and J. C. Sturm, Science 304, 987 (2004).

[3] M. Balvin, E. Sohn, T. Iracki, G. Drazer, and J. Frechette, Phys. Rev. Lett. 103, 078301 (2009).

[4] T. Kulrattanarak, R. van der Sman, C. Schron, and R. Boom, Adv. Colloid Interface Sci. 142, 53 (2008).

[5] M. MacDonald, G. Spalding, and K. Dholakia, Nature 426, 421 (2003).

[6] M. Z. Bazant and T. M. Squires, Phys. Rev. Lett. 92, 066101 (2004).

[7] T. M. Squires and M. Z. Bazant, J. Fluid Mech. 509, 217 (2004).

[8] H. Sugioka, Colloids Surf., A 376, 102 (2011).

[9] H. Sugioka, Phys. Rev. E 80, 016315 (2009).

[10] H. Sugioka, Phys. Rev. E 83, 056321 (2011). 